\title{
DEVELOPING AN ACTUAL VERY HIGH FREQUENCY ANTENNA USING GENETIC ALGORITHMS
}

\author{
Aatish Gandotra ${ }^{1}$, Tejaswi Singh ${ }^{2}$ \\ ${ }^{1}$ Student, Electronics and Communication Engineering, Guru Tegh Bahadur Institute of Technology, Delhi, India \\ ${ }^{2}$ Student, Computer Science Engineering, Guru Tegh Bahadur Institute of Technology, Delhi, India
}

\begin{abstract}
Antenna for the $88-108 \mathrm{MHz}$ Very high frequency (VHF) broadcast audio frequency-modulation (FM) band. The antenna is intended tofit in the flat area inside the head-band of an over ear hearing-protector headset. The space for the antenna is limited by an existing head-band design, where the unused internal area is the space studied in this thesis.

A genetic algorithm is described for the multiple objective optimization of the antenna matching and radiation pattern optimization. The results of multiple genetic algorithm evaluations are described, and possible further improvements outlined. Progress is made on the development of the antenna. The antenna radiation pattern is evolved in desirable way, but a difficulty in solving the antenna matching problem is identified. Research for resolving the antenna matching problem is described in this paper
\end{abstract}

Keywords: Antenna, Modulation, Genetic Algorithm, Frequency, Head-Band, Very High Frequency Broadcast.

$* * *$

\section{INTRODUCTION}

currently there is an increasing trend in the electronics industry to move from products with functional protrusions, such as antennas and wires, to integrated designs which hide or remove these protrusions. This can most clearly be seen in the mobile handset industry, but the same move can be seen in otherfields, such as hand-held transceivers, headset products and hearing protectors. For instance, a typical mobile handset may previously have featured a protruding antenna, protruding buttons and wires for connecting a headset. In the recent years these parts have been replaced with fully integrated antennas, at touchscreens and wireless headsets. The main advantages of this are improved device reliability for the end user and increased design freedom during the device design phase. The move to integrated, protrusion-less devices provide many new and unique design challenges for these internal parts: internal antennas must still be able to transmit and receive, despite the new volume restrictions. Button functions must still be available and mechanically reliable. Headsets must be able to function despite the lack of wires, etc.

The antenna described in the present work was conceived as a potential way for a generic FM-radio hearing protector to move to such an integrated, conformal antenna. Initially, the free volume between the head-band wires was identified as the most promising volume to house an antenna. A flexible printed circuit board was selected as a suitable manufacturing method for such an integrated antenna, being compatible with the mechanical stresses of product usage and promising low unit cost. The obvious design challenge posed is the shape and small volume of the available volume, in addition to the nearby metallic head-band wires. These limitations largely prohibit the use of traditional antenna designs and design methods.
In order to realize the antenna, an iterative method of antenna design using the genetic algorithm was identified as the best candidate for success. This paper aims at describing the work carried out in order to simulate this antenna, and to document the results. The antennas described were simulated using Numerical Electromagnetics Code 2 (NEC2). No hardware has been built to verify the simulations.

\section{BACKGROUND}

The primary motivation for this study is that existing antenna solutions, based on helical or wire whip designs, constitute a mechanical protrusion from the otherwise smooth features of a headset. This protrusion is a major source of mechanical fatigue problems. It is also an annoyance for the end user due to its ability to get tangled in foreign objects and as a source of conducted mechanical noises when it makes mechanical contact with foreign objects.

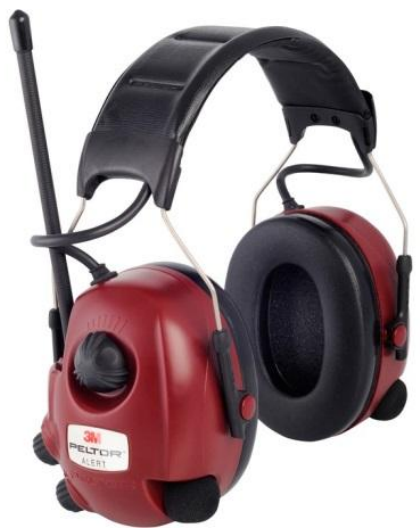

Figure 2.1: A typical headset of the type studied in this thesis

The type of headset targeted in this study, as shown in figure 2.1 , is typically used in an industrial or semi-industrial 
setting, while the end user is carrying out normal work. This implies that a cylindrical radiation pattern is desired, as the received signal strength should be stable while working facing different directions. It also places high demands on antenna efficiency and radio receiver sensitivity, as he use in industrial or semi-industrial settings commonly involve severe multiple-path fading situations.

\subsection{VHF Broadcast FM Radio}

The international VHF broadcast FM radio band of interest covers the frequencies $88-108 \mathrm{MHz}$ 's This is the frequency band used by the majority of nations, with the exception of Japan and some former Soviet republics[11]. Most of the former Soviet republics have, or are in the process of converting to the international VHF broadcast band. Table 2.1 outlines the basic parameters of the VHF FM broadcast system.

\begin{tabular}{l|l|l} 
Frequency range & $88-108$ & $\mathrm{MHz}$ \\
Polarization & Vertical & \\
Channel allocation raster & 50 & $\mathrm{kHz}$ \\
Channel bandwidth & 200 & $\mathrm{kHz}$ \\
Modulation limit & $+/-75$ & $\mathrm{kHz}$ \\
Pre-emphasis & $75 / 50$ & $\mu \mathrm{s}$
\end{tabular}

\subsection{Existing Antenna Solutions}

Antennas on existing headsets are usually of the shortened helical or whip type, with the headset printed circuit boards forming the ground plane. A typical design has an antenna which is approximately $18 \mathrm{~cm}$ in length, and use a $5 \times 5 \mathrm{~cm}$ ground plane. At the frequencies of interest, it is expected that the size limitations of the ground plane pose a major limitation for total antenna efficiency. At the center frequency $100 \mathrm{MHz}$, the $5 \times 5 \mathrm{~cm}$ ground plane is approximately $0,016 \lambda$ ( $\lambda$ denotes wave-length). While a larger ground plane would be technically desirable, it is not practical for a reallife application. The ground plane overall size is limited by the cup size, which is ultimately determined by the size of human heads.

\subsection{Possible Flexible Antenna}

The mechanical opportunity for an antenna was identified as the space inside the head-band of the headset, from a practical point of view; an etched flexible printed circuit board (PCB) antenna hidden inside the head-band appears feasible.

While other antenna types could be considered, for example a conformal antenna embedded in the ear-cup surface, the flexible-PCB proves several advantages over other types. These include a higher dimensional accuracy, ability to implement any conceivable two-dimensional structure, good mechanical flexibility and low cost. Since the final antenna design is unknown at the onset of work, it was decided that the flexible printed circuit antenna should be the type studied in this thesis.

The choice of a flexible printed-circuit defines the surface of the simulation output-space as the surface which may be covered by the flexible-PCB. It should be noted that in order to simplify the simulation, the carrier substrate for the flexible-PCB is not included in this simulations. Since the carrier substrate consists of a very thin layer of nonconductive dielectric it is believed that this is an acceptable simplification.

The flexible-PCB considered consists of essentially two layers omitting surface treatments, binders etc: an upper copper layer and a flexible carrier substrate material made of polyamide plastic. The copper is approximately $0.04 \mathrm{~mm}$ thick, while the thickness of the base substrate is approximately $0.3 \mathrm{~mm}$ [12]. A typical exible PCB is shown in figure 2.2. During theproduction process of a flexible PCB, the top layer is etched away using a photo-lithographic process, leaving a conductive copper pattern matching that of the designed artwork. Since the copper layer is very thin, and the polyamide is exible, the resulting PCB ishighly flexible. A bend radius down to $5 \mathrm{~mm}$ can typically be accepted without severing the copper, even in extended fatiguetesting situations [12].

This type of flexible-pcb therefore lends itself well to our planned antenna. The exibility allows for easy installationduring headset production, is possible to hide within existing structures, and allows for flexing of the entire antenna when the user isfitting the headset.

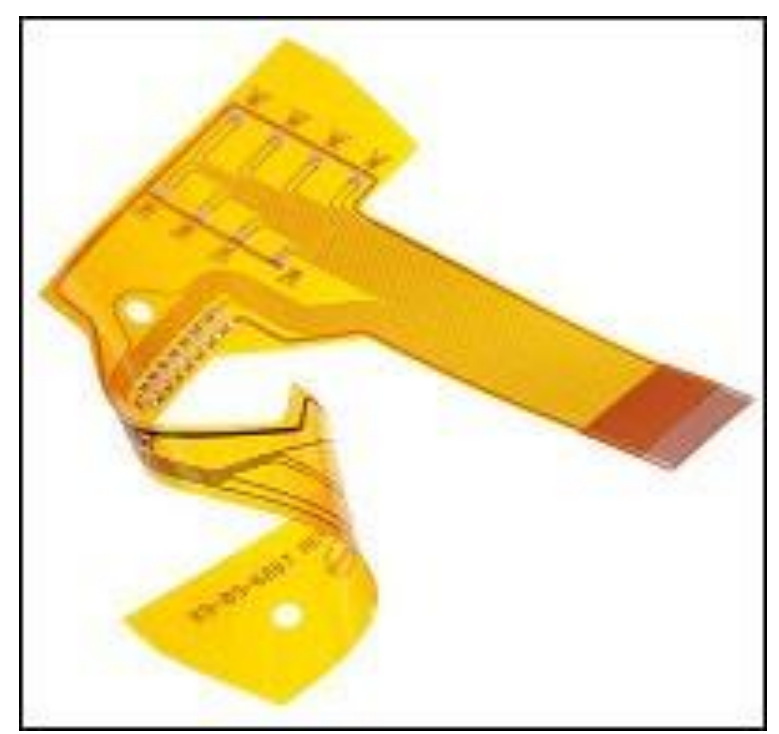

Figure 2.2: A typical flexible-pcb

\subsection{Existing headband}

The existing head-band studied in this thesis is based on two stainless steel metal spring wires which constitute the front and back main members of the head-band. The central part between the two wires are traditionally separated by non-conductive plastic, and constitute the space where the antenna elements may be placed. Due to the close proximity to the existing antenna - approximately $5 \mathrm{~cm}$ or 0 , $016 \lambda$ - the wires are expected to form a part of the existing radiating structure. Due to their placement, they are expected to primarily impair the radiation pattern symmetry, although this has not been verified by measurements. See

Figure 2.1 and figure 4.2 


\subsection{Radiation Pattern}

The antenna in this application is head-worn and used during normal work functions without regard to the placement or direction of the transmitter. It therefore follows that the primary requirement for the antenna radiation pattern is that it should be circular or close to circular in azimuth plane. Practical experience shows that a small deviation from the ideal circular pattern may be tolerated. Deep notches in the radiation pattern are highly undesirable, as the end user is prone to notice and experience and be annoyed by these regularly during the work day. The elevation pattern component variation is less critical, since a typical user tend to keep their heads nominally horizontal for most of the day. A dipole antenna radiation pattern, circular in azimuth and with deep notches only in the nadir and zenith location is therefore very suitable in this application.

\subsection{Antenna feed}

As the antenna is connected to the receiver circuit placed in the right hand cup of the headset, it is desirable to have the antenna feed point as close to this as possible. The preferred method to connect the antenna to the receiver circuit is by soldering a short coaxial cable directly to the feed point on the antenna. For cost reasons, the antenna feed coax should be kept as short as possible.

\subsection{Design Target}

Given the above details, the following design goals are identified 2.2

Frequency range $\quad 88-108 \mathrm{MHz}$

Omnidirectional gain $>2 \mathrm{~dB}$

Directionality tolerance $0.3 \mathrm{~dB}$

VSWR $<5$

Polarization vertical, nominal

Antenna feed system Soldered coax cable on right extreme of antenna. Manufacturing method Etched exible printed circuit board.

\section{THEORY}

\subsection{Relevant Antenna Theory}

A short summary of the relevant fields of antenna theory is provided below.

\subsubsection{Far Field Theory}

In the very simplest, theoretical antenna cases, the far field radiation pattern can be calculated analytically [13]. This can then be used for evaluating the performance of the antenna directly, which is useful for explaining the relevant antenna far field theory. The description below follows the outline provided by Cheng [13].

A simple Herzian dipole consists of a short conductive wire terminated with two small conductive wires. Assume a

sinusoidal current flows in the wire:
$\mathrm{i}(\mathrm{t})=\mathrm{I} \cos (\omega \mathrm{t})(3.1)$

Since the current diminishes at the end of the wire, charges must build up there, and we canwrite the relation between charge and current as

$$
\mathrm{i}(\mathrm{t})= \pm \mathrm{dq}(\mathrm{t}) / \mathrm{d}(\mathrm{t})
$$

Using the magnetic vector potential $\mathrm{A}$, from $\mathrm{B}=\nabla \times \mathrm{A}$, it can be shown that

$A=a^{\mathrm{z}}\left(\mu_{0} \operatorname{Id} \ell\right) / 4 \pi\left(e^{-j \beta R R}\right)$

From the above equations, it is possible to identify some basic properties of the results in the far field, for a bound source:

1. $\mathrm{E}_{\theta}$ and $\mathrm{H}_{\theta}$ are in space quadrature and in phase

2. The ratio $\left|E_{\theta}\right| /\left|H_{\theta}\right|=\eta_{0}$ is constant. This constant corresponds to the impedance of the medium

3. The magnitude varies inversely proportional with the distance to the antenna. The phase of $\mathrm{E}_{\theta}$ and $\mathrm{H}_{\theta}$ is a periodic function of the distance with a period that is the wavelength, $\lambda=\mathrm{c} / \mathrm{f}$

Also it is possible to plot the radiation pattern of this antenna. The far field radiation pattern describes the relative far field strength as a function of direction, on a fixed direction from the antenna. The radiation pattern plot is shown in figure below.

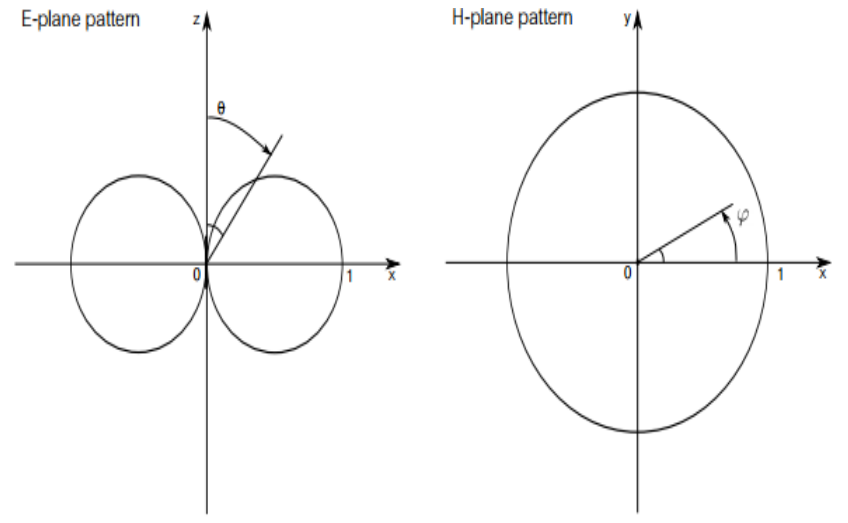

Figure 3.1: Radiation pattern for a herzian dipole, $\mathrm{H}$ and $\mathrm{E}$ plane

In order to relate this result to the generic case, it is necessary to consider the case of a real, linear dipole. Relating the result to a real, finite dipole, presents a mathematical challenge.

While the problem is largely the same, the determination of the exact current distribution on the antenna is a di-cult boundary-value problem. An obvious observation is that the current must be zero at the ends of the antenna ends, and finite in the feed point, but this unfortunately does not provide any major breakthrough in solving the current distribution.

An analytical equation still leads to an integral equation where the current distribution is unknown under the integral.

\subsubsection{Polarization}

The polarization of a uniform planar wave, for example emanating from a dipole antenna, describes the time-varying 
behavior of electric field intensity of a vector at any point in space. When the $\mathrm{E}$ vector of a plane wave, is fixed in the $\mathrm{X}$ direction the wave is said to be linearly polarized in the $\mathrm{x}$ direction. Similarly a plane wave may be linearly polarized along the other unit axes and said to exhibit the corresponding linear polarization. In cases where the direction of the $\mathrm{E}$ vector of a plane wave changes with time, the plane wave is said to exhibit nonlinear polarization, either elliptical or circular.

It can be shown that this case, the plane wave may be described by a superposition of two linear polarized plane waves, one linearly polarized along the x-direction and one linearly polarized along the $y$-direction. In the case where the $\mathrm{x}$-direction and $\mathrm{y}$-direction polarized plane waves have the same linear magnitude, and are in phase quadrature, the composite field is said to exhibit circular polarization. If this is not the case, the plane wave is said to exhibit elliptic polarization. It can be shown that if the transmitting and receiving antenna does not share the same polarization, path losses will increase by a factor depending on the angle of mismatch between the two antennas [13].

This factor, known as Polarization Loss Factor, is defined as

$$
\mathrm{P} L F=\cos ^{2} \phi(3.3)
$$

Where PLF is the linear loss factor due to the polarization mismatch, and $\phi$ is the misalignment angle. Studying equation 3.12, it can be shown that a small misalignment has little effect, but if the polarization misalignment is 90 , no power at all is transmitted between the two antennas.

The VHF FM broadcast radio system employs a linear vertical polarization. Historically, this was chosen because vertical polarization provides the minimal propagation attenuation in most terrestrial line-of-sight or near-line-ofsight situations at these frequencies [14], and because it usually simplifies antenna installations when using simple dipole or ground-plane antennas.

Studies of real-world propagation effects show that a clean polarization may degrade into a set of superimposed polarized fields, due to reflections on nearby metal objects. A study carried out by Shrauger and Taylor have shown that, in a real terrestrial situation, in our band of interest the nominal received polarization often greatly differ from the nominal vertical transmitted polarization[14]. This is the primary reason for considering the polarization a Secondary property of this antenna

\subsubsection{Impedance}

The input impedance of an antenna is defined as the ratio of voltage to current $\mathrm{Z}=\mathrm{U} / \mathrm{I}$, as presented to the antenna feed structure. The antenna impedance will depend on many parameters of the antenna, primarily mechanical design and measured frequency [13]. Antenna impedance is - in this case - important primarily in order to be able transfer the maximum of the received energy to the radio receiver circuit, as described in the matching section below. Antennas for VHF broadcast receivers are traditionally designed to have a 50 or $75 \mathrm{Ohm}$. In order to be able to compareand ultimately measure different antenna systems, it is therefore desirable to adhere to this convention

\subsubsection{Radiation Resistance}

A measure of the amount of power radiated by an antenna is the antenna radiation resistance; Rr. is theoretical resistance that would dissipate power equal to the radiated power when the current in the resistance is equal to the maximum current along the antenna. It is therefore desirable for an antenna to have a high radiation resistance.

\subsubsection{Antenna Matching}

Antenna matching is the measure of how well the impedance of the antenna matches the impedance of the load circuit. When these impedances are equal, the maximum power can be transferred from load to antenna and vice versa. In this case the antenna and load are perfectly matched. When the impedances are non-equal, part of the available power is lost by being reflected at the connection interface.

\subsubsection{Electrically small antennas}

An antenna which is mechanically considerably smaller than the wavelength on which is it designed to operate is said to be electrically small. Such antennas exhibit considerable differences compared to a larger antenna. There has been considerable work done on studying the properties of electrically small antennas, and ascertaining what exactly constitutes an electrically small antenna. Initial work was carried by Wheeler [8], Chu [7]. Refined by Hansen [15], and more recently refined by Gustafson et al. for thin antennas [9]. The initial, still commonly used, definition of an electrically small antenna is an antenna with volume $\mathrm{V}$ smaller than $(2 * \pi * \mathrm{r}) / \lambda[8]$. Although this is clearly a nonideal metric for our strip shaped antenna, it is still useful in order to conclude that the antenna is indeed electrically small, since $\lambda=3.4 \ldots 2.8$ meters and $r=0.20$ meters. For a small antenna, the background work leads us to expect the following properties

\section{High current densities}

2. Difficulty in matching the antenna

3. A very small radiation aperture

\subsection{The NEC2 program}

The NEC2 program uses a combination of the electric field integral equation (EIFE) and magnetic field integral equation (MFIE), as a basis for calculating the current distribution on antennas [16]. From the current distribution, it is possible to calculate both the impedance of the antenna and the radiation pattern. EFIE is used for thin-wire structures, and MFIE is used when calculating voluminous structures.

They are combined into a hybrid equation, and this is solved numerically using the method of moments, by describing each antenna segment by a number of base functions. The current on wires and structures are then described in matrix form.

The solution matrix is consequently solved by Gaussian elimination. From this it is possible to and the requested antenna impedance and the far field radiation pattern. 


\subsubsection{NEC2 limitations}

The NEC2 program has several known limitations, such as its limited support for dielectric volumes, and the difficulty in accurately simulating complex three-dimensional shapes. These are of little consequence to the problem at hand, which is entirely modeled as separate wires.

However, a less obvious limitation clearly has implications to this problem: Due to the design assumption of each wire segment being either end fed or open ended, an antenna where segments intersect between end-points cannot be simulated without exception.

This is normally not a problem since segments can simply be split up so that they either begin or end in intersections, thus avoiding violation of the model.

In this case though, that limitation presents a problem as antenna segments may or may not cross depending entirely on the result of the genetic algorithm convergence. As this is not exception handled in the current genetic algorithm, it casts some doubt over the validity of the simulation results in the cases where this happens. Currently, solutions could be screened manually for violations of this NEC2 limitation, as it is not caught automatically by the genetic algorithm.

\subsection{Genetic Algorithm Theory}

A genetic algorithm is an specialized optimization method which mimics the process of natural selection [17] for solving a specific problem. This method was pioneered by Holland in 1975 [18]. By this method, genetic algorithms are able to search a multi-dimensional solution space and find a solution reasonably efficiently. Genetic algorithms are therefore well suited to the area of antenna design, where the search space is wide and multi-dimensional but straight-forward to simulate. The two key features of the algorithm are

- A basic genome, through which a large set of potential solutions for the problem at hand may be described.

- A fitness function, through which individual solutions are evaluated and chosen for generating new solution.

The algorithm works by setting up a number of candidate solutions, described by the genome. Each solution is then simulated and evaluated by the fitness function. The top ranking solutions are then chosen for reproduction into a new generation, where the best solutions are combined in order to find solutions which may be closer to the design target. The process is repeated until a desirable solution is found [19].

Each population iteration is referred to as a generation, and solutions are referred to as parents and children in analogy with the process of natural selection. In order to maintain the progress of the algorithm, it is essential that the basic genome is able to describe the parameters relevant to the problem at hand, and that those parameters may be transferred from parent to child.

\subsubsection{Chromosome Representation}

The chromosome is the solution vector used for each individual solution candidate. Each parameter in the chromosome is referred to as a gene.
The chromosome representation used in the genome is highly influential on the performance of the genetic algorithm. A too constrained chromosome design will not allow the algorithm to find the necessary solutions, but an overly exible design may on the other hand slow down convergence or prevent convergence altogether [19]. Apart from exibility, the chromosome design should also be able to relate to the problem at hand in a relevant way, so that relevant properties of the solution may be transferred from parent to child during cross-over between different parents, in order for desired solutions to be able to propagate between generations.

\section{Implementation}

A multi-objective genetic algorithm was implemented in Matlab, with the NEC2 program used as the simulation backend. Matlab is well suited to genetic algorithm tracking and development, as several plotting and debugging tools needed are readily available. Apart from that, almost any programming language could have been employed for the same purpose.

The NEC2 simulation software is a critical part of this thesis, as it is well proven and easy to interface from an automated algorithm. Its method-of-moments simulation foundation, while unsuitable for certain types of antennas, is well suited to the wire-type antennas studied in this thesis.

\subsection{Genetic Algorithm}

The genetic algorithm largely follows the theoretical description as outlined. The genetic algorithm is implemented in Matlab, and loops through each individual in each generation until specific termination criteria are met. The flow of the implemented algorithm is shown in figure 4.1. In this thesis the termination criteria isis based on numbers of generations.

\subsection{Mechanical model}

The mechanical model for the antenna simulation consists of two fundamental parts. An overview of the mechanical model is given in figure 4.2. The fixed part is made up of the static, pre-existing, structure. This is two wires of $2 \mathrm{~mm}$ stainless steel. The shape of these wires is set by mechanical requirements for the headset. They provide support for the headset cups and give the necessary holding force for the earcups. The exact dimensions for the fixed part are based on measurements on real headsets.

The second part is the variable antenna surface, where the genetic antenna is free to place wires to meet it optimization targets. For simulation purposes the antenna elements are considered to be thin wires segments, $1 \mathrm{~mm}$ in diameter. In a real product they would be at traces, etched on the exible PCB.

A fixed diameter is chosen in order to make sure no wire diameters may violate the manufacturing possibilities for the flexible PCB.

The active antenna surface part is modeled as a thin section of a cylindrical surface, where the antenna genetic algorithm places a number of elements, the first being the fed element. 
The variable antenna surface is highlighted in blue in figure 4.2 .

The coordinate system used for this model is a right-handed Cartesian coordinate system, as used by NEC2. Specifically, the end-user faces along the positive-Y axis, the end-user leftright axis is $X$, while the user up direction coincides with the $\mathrm{Z}$ axis.
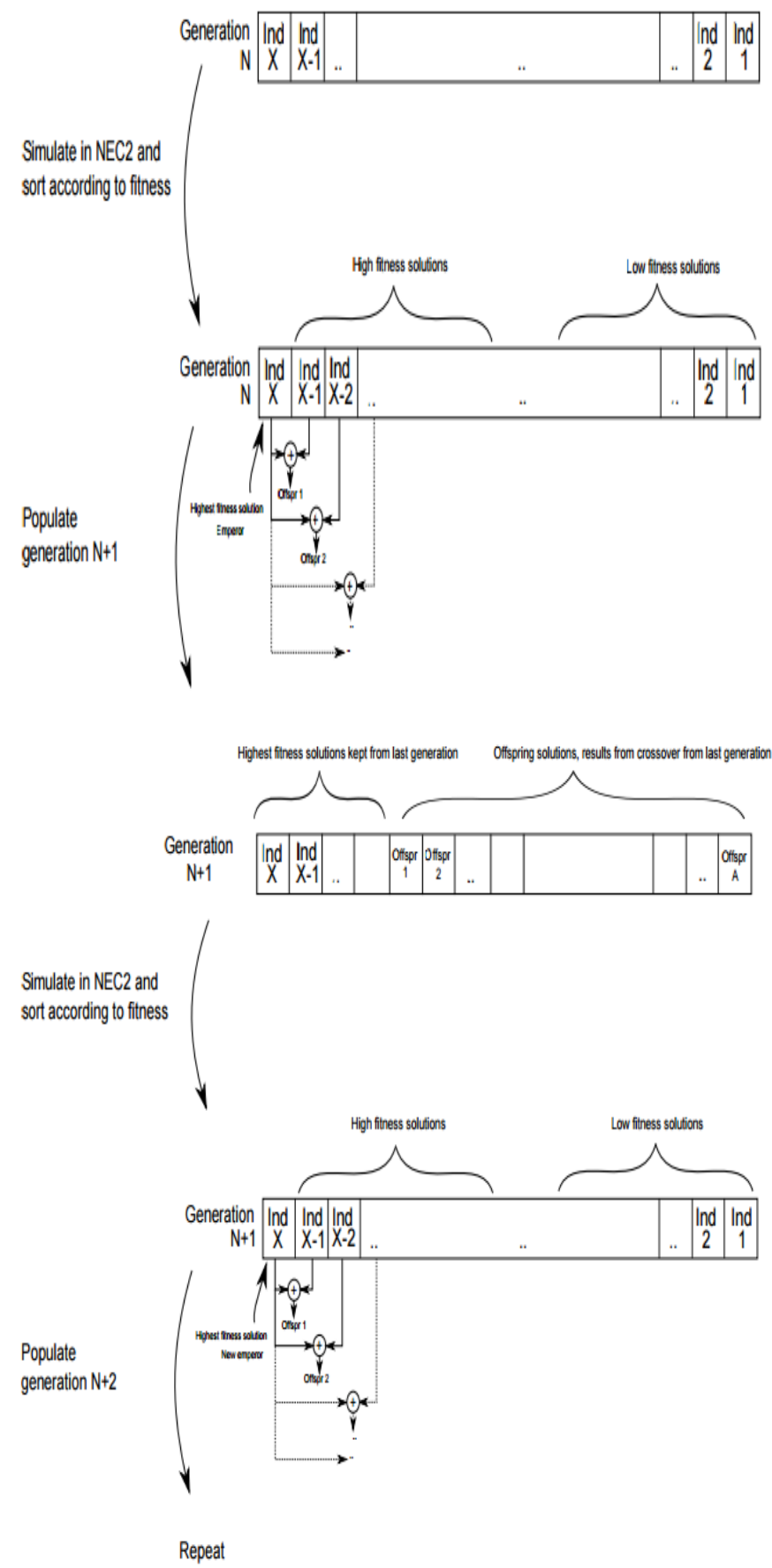

Figure 4.1: Genetic algorithm, generation progress
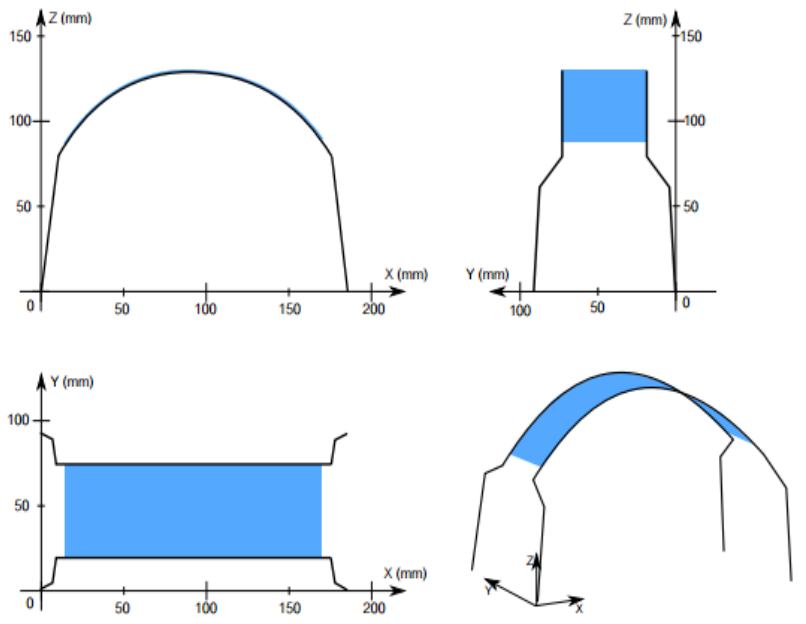

Figure 4.2: Head-band model base, space for antenna elements in blue

\begin{tabular}{|c|c|c|c|c|}
\hline Element & \multicolumn{2}{|c|}{ Start } & \multicolumn{2}{|c|}{ Stop } \\
& $\mathrm{X}$ & $\mathrm{Y}$ & $\mathrm{X}$ & $\mathrm{Y}$ \\
\hline 1 & 0 & 0 & 0.1 & 0.1 \\
\hline 2 & 0.1 & 0.1 & $0 . .1$ & $0 . .1$ \\
\hline 3 & 0.1 & 0.1 & 0.1 & $0 . .1$ \\
\hline 4 & 0.1 & $0 . .1$ & $0 . .1$ & 0.1 \\
\hline 5 & 0.1 & 0.1 & 0.1 & $0 . .1$ \\
\hline
\end{tabular}

Figure 4.3: Chromosome representation for each solution candidate

\subsection{Chromosome Design}

For this thesis a Cartesian coordinate system genome was chosen, with five movable wire segments. The genome space spans a $[0: 0] \ldots[1: 1]$ unity X-Y coordinate space, where each wire start and stop coordinates is represented as $\mathrm{X}-\mathrm{Y}$ pairs. The number of wires may be varied, and the initial number was arrived on after initial manual tests suggested that five segments provided sufficient freedom of design. All wires are fixed to be $1 \mathrm{~mm}$ in diameter, which is not adjustable by the genome.

This straight-forward design makes debugging and rangechecking practical and easy to verify. The major drawback is it may be non-ideal from an optimization point of view, since rotational translation is not possible in a simple genome cross-over scheme. This is left as the subject for a further study, as a polar coordinate representation would be considerably more complex to analyze from a theoretical algorithm point of view.

\subsection{Antenna Feed Point}

The antenna feed-point is fixed by the algorithm to the center of the first element in the genome. The starting coordinate of this first element is forced to genome coordinates $\mathrm{x}, \mathrm{y}=[0$, $0]$. The other coordinates of this element is subject to genetic algorithm cross-over and noise like the other elements in the genome. This ensures that while algorithm is able to move the 
free segment end, other end is fixed in the lower right corner. The center feed point is therefore kept reasonably close to the desired location.

\subsection{Symmetry}

Theoretically the antenna is fully symmetrical along the frontto-back axis. This may havebeen employed to simplify the genome, but this would have precluded the use of an asymmetric left-side coax feed, as specified in the design target. It was also believed that a future addition to the simulation would be the addition of the asymmetrical left and right handcircuit boards, which further removes symmetry from the problem. Therefore the current genome design does not employ any simplification due to symmetry.

\subsection{Coordinate Projection}

Since the genome design is based on a Cartesian coordinate space, as is the output simulationthree dimensional (3D) spaces, it is possible to map genome space directly to $3 \mathrm{D}$ space with asimple coordinate mapping. A study of the mechanical design gives the following coordinateprojection. The mapping function is visualized in figure 4.4.
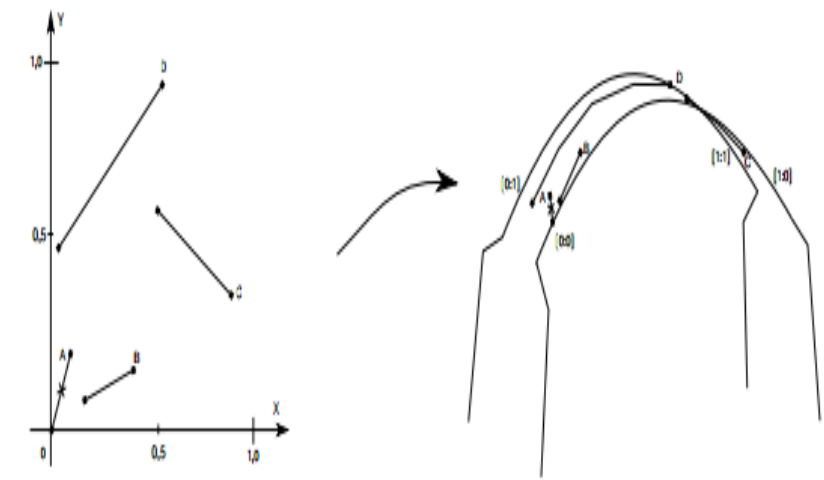

Figure 4.4: Coordinate mapping. The antenna feed point is marked with an $\mathrm{X}$

For any short wire segment of the output antenna, a straight line between the mapped beginning and end coordinates is sufficient. For wire segments with larger x-coordinate separation, a segmentation scheme is needed to keep the antenna segment sufficiently in-plane due to the curved nature of the output $3 \mathrm{D}$ space. In the case where a wire spans a longer section of the curved plane, a segmentation function is used to keep the wire segment sufficiently in-plane.

\subsection{Solution Mating And Selection Process}

The solution mating and the selection process determine a genetic algorithm convergence performance [19]. It is generally desirable to select the top ranking solutions for mating into new solutions. There is a clear trade of between focusing solely on the top individuals and maintaining the genetic diversity of the rest of the generation with lower rankings. A too tight focus on top individuals will tend to converge to a solution relatively quick, but may employ a poor exploration of the solution space. A too high focus on the lower rating solutions may, on the other hand, completely fail to converge on an acceptable solution or cause very slow progress. There are several mating schemes that have been successfully employed in similar cases, such as best-matesworst, adjacent-fitness-pairing and emperor-selective. In a study of mating schemes carried out by B.K Yeo in 1999, the emperor-selective was found to give the best solution convergence [20], and this method was therefore selected. The emperor-selective matching scheme starts by identifying the highest ranking solution for each generation. This solution, dubbed the emperor, is then mated with the remaining top-ranking solutions to produce the desired number of ospring solutions. A certain amount of overlap between generations is usually also employed, where a percentage of the previous generation top-ranking individuals are kept on to the next generation. This is done to maintain genetic diversity and ensure that the solution set does not degrade if all offspring in a generation should rank lower than the previous generation. In this thesis an overlap of $10 \%$ is used, as it was found to give acceptable progress results.

\subsection{Solution Cross-Over Function}

The cross-over function, used for producing ospring solutions, selects antenna segments from either parent by the following random selection:

- By a $31 \%$ probability, the wire coordinates are copied from the first parent

- By a $31 \%$ probability, the wire coordinates are copied from the second parent

- By a $31 \%$ probability the wire is generated as a mean between the coordinates used by the rst and second parent. • By a $7 \%$ probability, the wire is subject to a randomization, where the coordinates are replaced with a $0 . .1$ square probability distribution random coordinate regardless of parent coordinates.

\subsection{Fitness Evaluation}

In a multi-objective genetic algorithm, fitness evaluation is central to the progress of the optimization. In this thesis, the two fitness determining factors are the radiation pattern shape and the impedance matching of the antenna. These factors are evaluated separately as two parameters, pattern fitness $P_{f}$ and impedance fitness $Z_{f}$. The overall fitness for each solution is evaluated as

$$
F=P_{f}+Z_{f}
$$

\subsection{Pattern Fitness}

As stated above, the radiation pattern should ideally be completely rotationally symmetrical, vertically polarized, and exhibit some gain. To rate the fitness, each solution vertical gain $\mathrm{Av}[\mathrm{dBi}]$ is evaluated at center frequency $\lambda_{0}$ in

the polar $\Phi$ plane, maintaining $\Theta=90$. This vertical gain data set is available directly from the NEC simulation. For each solution: 


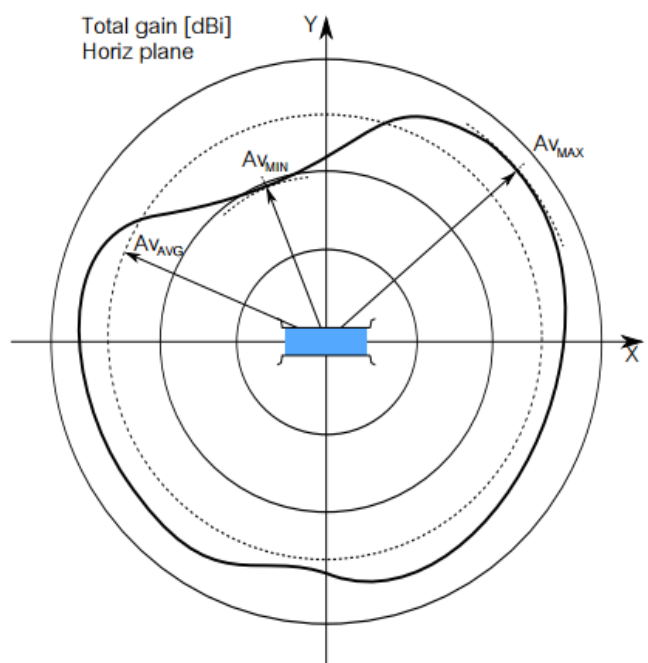

Figure 4.5: $\Phi$ plane vertical gain $A v, \Theta=90$ : Fitness rating of radiation pattern

the maximum gain $A v_{\max }$, minimum gain $A v_{\text {min }}$ is found, and the average gain $A v_{\text {min }}$ is calculated. A typical pattern is shown in figure 4.5.

\subsection{Population Size and Termination Criteria}

In any genetic algorithm, there is an obvious tradeoff between evolution speed and search width. With a small population size, each generation will obviously be simulated quicker than a large population, since the total time to simulate each generation

$$
\mathrm{T}_{\text {generation }}={ }_{\mathrm{T} \text { individual }} * \mathrm{~N}_{\text {individuals }} \text {. }
$$

The solutions in a genetic algorithm with a small population size will therefore also tend to evolve quicker than a large one. A small population size may not efficiently explore the given solution space. Such an algorithm runs the risk of converging on a sub-optimal local solution, taking an excessive number of generations to converge on a solution or being unable to converge on a solution at all[6].

The initial compromise was therefore set at 50 individuals per generation. This was found to be the largest population size where algorithm development was still practical. At larger generation sizes, the time to simulate each generation considerably hindered algorithm development. As a validation of the 50 individual generation size, tests were carried out using larger generation sizes. This did not notably change the evolution progress, apart from reducing algorithm progress speed, and the choice was therefore deemed acceptable. As termination criteria, the fixed number of generations' method was chosen. While this is known to be a suboptimal method from a computational efficiency point of view, it was found to be acceptable for this problem.

\subsection{Tracing And Debugging Tools}

As the run-time for this genetic algorithm is in the multi-hour range, direct feedback of the progress, as well as methods to perform post-run analysis is needed. Several methods for assessing progress were developed. The most obvious result to plot is the best-individual performance, as a function of generations passed. This gives a clear feedback of the algorithm evolution progress. Also, impedance matching ratings and pattern rating progress may be 21 plotted in the same graph, in order for their individual progress to be assessed.

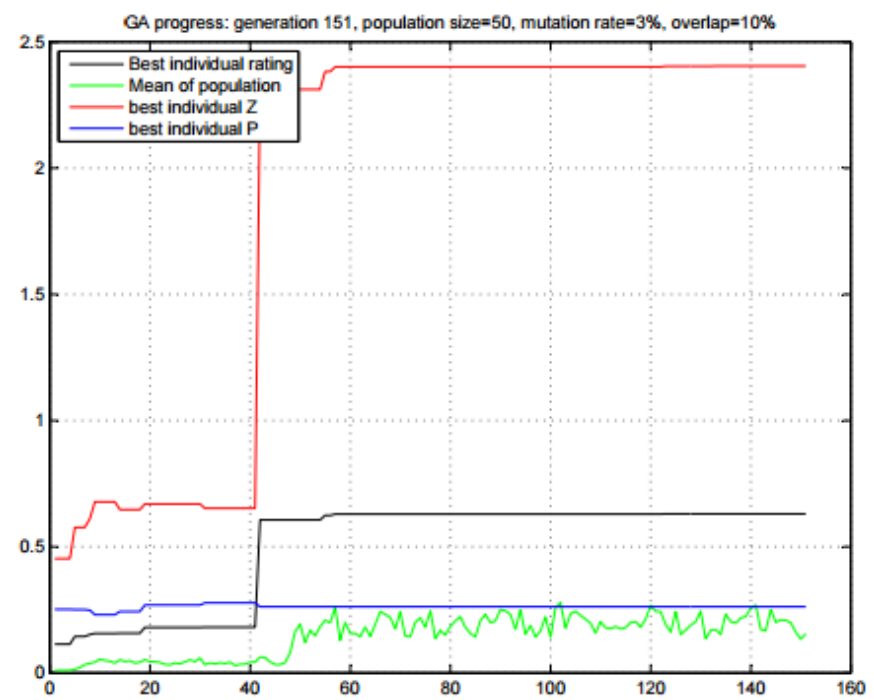

Figure 4.7: Typical progress plot, 150 generations elapsed

The optional visualization of each solution output data is also employed. This allows the fitness rating functions, and genome antenna wire design to be assessed in real-time. The visualization makes it possible to catch unexpected behavior of the fitness functions, or their reaction to unexpected input from the NEC simulations. As the genetic algorithm will converge on whatever solutions that give the highest ranking, it is very important that the rating functions be carefully debugged. Without these visualization functions, these core functions would be very difficult to debug. As the genetic algorithm convergence is highly nonlinear, it is also desirable to provide means for detailed post-analysis of each run, so that what appears to be undesirable behavior may be analyzed and rectied. As an analogy to natural evolution, the genetic algorithm is therefore fitted with means to create fossil records at certain intervals. These are complete records of a generation, stored to separate each 50 generations. After each run a complete fossil strata is therefore present for analysis, should this be necessary. After the genetic algorithm terminates, a 3D surface is plotted which displays individual rating as a function of generations passed. This allows the total convergence progress to be assessed.

\subsection{Noise Between Generations}

In order to keep the genetic algorithm from converging on a solution which relies too heavily on close tolerances, a certain amount of noise is added between generations. This makes sure that solutions may not require unrealistic manufacturing tolerances. It also avoids convergence on any mathematical Singularities, should they arise. A random noise with continuous uniform distribution and with max amplitude of $0.01 \mathrm{~mm}$ is added to all coordinates of generated offspring solutions. A uniform distribution is chosen as a first-order approximation of the expected error distribution in production 
of a printed antenna. This is caused by a production process where any position within a tight tolerance is accepted, and any outliers are discarded.

\section{RESULTS}

\subsection{Genetic Algorithm}

The genetic algorithm appears to be functioning as intended. Antennas are produced within the given mechanical constraint. There is a clear progress of evolution where initial solutions can be clearly seen to replace their previous, less fit, predecessors. As the algorithm is started with a different random seed for each run, each run is dierent. All runs show clear progress toward the intended target. It is clear that the impedance problem is not handled nearly as well as the radiation pattern problem.

\subsubsection{Initial Solutions}

The top-ranking solutions of the very rst generations generally exhibit very poor matching. At this stage, a solution with acceptable, or close-to acceptable omnidirectionality may often be found. Note that the antenna is acceptably omnidirectional, but that the average gain is only around $1.3 \mathrm{dBi}$, varying around $0.5 \mathrm{~dB}$. From the $3 \mathrm{D}$ diagram it is noted that this is caused by the radiation pattern being frontto-back tilted approximately 40 degrees.

\subsubsection{Late Solutions}

The top-ranking solutions of the final generation generally still exhibit very poor matching, with little or no improvement from the initial solutions. At this stage, however, the solution usually exhibits very good omnidirectionality. This now exhibits $2.2 \mathrm{dBi}$ gain, within $\mathrm{a}+/-0.01 \mathrm{~dB}$ tolerance.

\subsection{OBJECTIVES}

\subsubsection{Radiation Pattern}

Radiation pattern optimization works well. The resulting radiation pattern show a high degree of omnidirectionality, and would likely to perform well in a real-world application. The demonstrated omnidirectionality and relative gain is equal to, or better then what can be expected from a traditional whip-based antenna design in this type of application. This objective is considered met.

\subsubsection{Antenna matching}

Antenna matching has proven di-cult to archive, and the matching goals have not been met. The resulting antennas show too poor matching to be usable in a real product. While it is clear that the genetic algorithm is able to reward solutions with improved matching performance, these solutions do not seem to propagate well through the genome. With the dimensions for our antenna at hand, $\mathrm{R}=0.2 \mathrm{~m}$, and $\lambda=3 \mathrm{~m}$ we get $\mathrm{Rr}=0,035 \mathrm{Ohm}$. This is a very small value, and we may therefore expect that such a small dipole would be a very poor radiator. It should be noted that Herzian dipoles, when $\mathrm{dl} \ll \lambda$, but it gives us a reasonable indication of performance. By increasing the number of segment in the algorithm, and hence the antenna length we expect that the impedance should improve slightly since short antennas have low impedance. A system that rewards useful SWRs at any frequency in the band would also likely improve the algorithms development of matching.

\section{CONCLUSIONS}

A multi-objective genetic algorithm for creating an omnidirectional antenna inside the headband of an existing headset is demonstrated. The algorithm is demonstrated to show convergence towards the desired solutions. Typical average gains are shown to start out at $1.3 \mathrm{dBi}$, with a rotational variation of $0.5 \mathrm{~dB}$ and end up at $2.2 \mathrm{dBi}$ with a rotational variation of 0.01 . This shows that the algorithm is able to generate antennas with a directional radiation pattern in close accordance to the design target. The algorithm is not able to solve the problem of matching the antenna; the possible reasons for this are discussed in the following chapter. Polarization optimization is not included but could be added as a secondary requirement. Several possible additions to the original scope of the thesis work have been identified.

\section{PRACTICAL LIMITATIONS}

Some known limitations in this work are listed below

- Intersected segments are present, even though this violates the fundamental NEC2 assumptions. A way to avoid this would be to replace NEC2 with simulation software which does not suffer from this limitation.

- A model for the end-user head is not included. This is due to the fact that NEC2 it not able to handle dielectric bodies well, and it was therefore decided to omit the head from the model. The antenna designs in this thesis could be easily tested in real-life without an end-user head, in order to verify the simulation accuracy. The presence of a real head is not expected to invalidate any of the prior results.

- The printed circuit boards for the headset electronics are also not included. These could easily be simulated by NEC2, if so desired, but it was believed that they could safely be omitted in order to speed up the simulations.

Like with the head model, an antenna could be tested in reallife without printed circuit boards, in order to validate the antenna simulation results.

\section{IDEAS FOR FUTURE IMPROVEMENTS}

During the development of this thesis, some ideas for future developments have been noted. These have not been possible to investigate during the thesis work, but could be the subject of future studies.

- A thorough analysis of the problem from a theoretical point of view could be conducted, extending from the basic review above. With the recent results provided by Gustafsson et. al., it should be able to theoretically establish the fundamental limitations present for a thin antenna of this shape [9]. This may establish if the 
problems encountered are due to fundamental physics or merely technical limitations of the algorithm. A careful study of the recent advances in this field may additionally provide further insight into possible ways to avoid the most severe limitations, and ultimately provide a more successful antenna design.

- A different genome which would allow complex structures to better transfer between solutions could be investigated. This should allow matching structures to better survive between generations and cross-overs and may also provide an improved stability of the matching solutions. At the same time a corresponding cross-over function could be investigated. A study could be conducted of the feasibility of different genomes and their cross-over functions described in recent literature, and their suitability to the problem on-hand. A basic literature study does not present a great deal on the subject which is directly applicable to this problem. The two-dimensional, small size, nature of the problem appears to make the subject relatively rare.

- A fitness rating rewarding vertical polarization could be added. This would allow for a shift towards vertical polarization in the final antennas, should the specification be changed to request this.

- The termination criteria could be updated with suitable fitness-based termination criteria, should the major obstacle of impedance fitness rating be overcome. This would allow the time to convergence to be assessed in greater detail and most likely result in a shorter average time to convergence [19].

- Finally, different weighting functions of the antenna fitness parameters could be considered. While the weighting functions have been the subject of considerable work in this study, without much progress, it cannot be completely ruled out that it may be possible to improve them further.

\section{REFERENCES}

[1] D. Marcano and F. Duran, "Synthesis of linear and planar arrays using genetic algorithms," in Electromagnetic Optimization by Genetic Algorithms, 1999, pp. 157-180.

[2] E. E. Altschuler and D. S. Linden, "Design of wire antennas using genetic algorithms," in Electromagnetic Optimization by Genetic Algorithms, 1999, pp. 211-248.

[3] D. S. Linden, "Rules of thumb for ga parameters, ga monitoring, ga parameter optimization, and enhancement of ga e-ciency," in Electromagnetic Optimization by Genetic Algorithms, 1999, pp. 67-94.

[4] E. E. Altshuler, "Electrically small self-resonant wire antennas optimized using a genetic algorithm," in IEEE transactions on antennas and propagation, vol. 50 no. 3, March

2002, pp. 297-300.

[5] A. S. James Dietrich, "Automating nec2 with matlab for antenna analysis and design," inProceedings of the 2002 Canadian Conference on Electrical and Computer Engineering, 2002, pp. 342-346.

[6] Y. R. Tsoy, "The inuence of population size and search time limit on genetic algorithm," in Proceedings KORUS 2003, vol. 7, June 2003, pp. 181-187.
[7] L. J. Chu, "Physical limitations of omni-directional antennas," in Applied physics, vol. 19, May 1948, pp. 11631175 .

[8] H. A. Wheeler, "Fundamental limitations of small antennas," in Proceedings of the I.R.E,vol. 35(12), December 1947, pp. 1479-1484.

[9] M. Gustafsson, C. Sohl, and G. Kristensson, "Illustrations of new physical bounds on linearly polarized antennas," in IEEE transactions on antennas and propagation, vol. 57 no. 5, May 2009, pp. 1319-1327.

[10] S. M. Jain, S. K. Koul, A. Basu, and M. P. Abegaonkar, "Recongurable conformalantenna array for non-rigid platforms," in Proceedings of Asia-Pacic Microwave Conference2010, 2010, pp. 20642067.

[11] I. T. Union, "Bs.450 : Transmission standards for fm sound broadcasting at vhf," 2001,http://www.itu.int/rec/RREC-BS.450-3-200111-I/en.

[12] C. Inc, "Castronic volume ex pcb," 2003-2007, castronic.com/pcb_volumeex.htm.

[13] D. K. Cheng, Field and Wave Electromagnetics. Addison Wesley, 1989. ISBN 0-201-52820-7

[14] N. K. Shrauger and K. L. Taylor, "Initial vhf propagation results using xeledop techniques and low antenna heights," 1966, www.dtic.mil/cgi-bin/GetTRDoc?AD=AD0653609.

[15] R. C. Hansen, "Fundamental limitations in antennas," in Proceedings of the IEEE, vol. 69(2), February 1981, pp. 170182.

[16] G. Burke and A. Poggio, "Numerical electromagnetics code (nec) - method of moments," 1981, part II: Program description - code.

[17] C. R. Darwin, On the origin of species by means of natural selection. London, JohnMurray, 1859.

[18] J. H. Holland, Adaptation in Natural and Artificial systems. University of Michiganpress, 1975. ISBN 9780262581110

[19] Y. Rahmat-Samii and E. Michielssen, Electromagnetic Optimization by Genetic Algorithms.Wiley, 1999. ISBN 0471-29545-0

[20] B. K. Yeo and Y. Lu, "Array failure correction with a genetic algorithm," in IEEEtransactions on antennas and propagation, vol. 47 no. 5, May 1999, pp. 823828.

\section{BIOGRAPHIES}

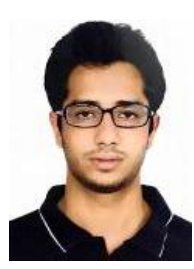

Aatish Gandotra, a student of Electronics and Communication Engineering in his final year with deep interest in telecommunication and networking.

Publications :- 1. Replication of attacks in wireless sensor network using NS2.

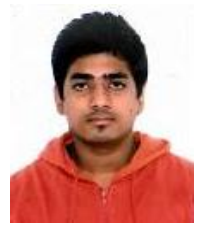

Tejaswi Singh, a student of computer science engineering in his final year with a knack for network security.

Publications :- 1. Replication of attacks in wireless sensor network using NS2. 